\title{
INTRODUCTION
}

\section{Hippocampal Place Fields: A Neural Code for Episodic Memory?}

For over a half of a century, neuroscientists speculated about the neurophysiological mechanisms that support hippocampal-dependent learning and memory. About 35 years ago, O'Keefe and Dostrovsky (1971) published a report that ultimately resulted in a paradigm shift (Kuhn, 1970) in this field. Their report showed that hippocampal pyramidal neurons selectively discharge as animals traverse a circumscribed location within its environment. This was an astounding finding not only because of the surprising clarity of the neural signal from a brain structure thought to be involved in complex forms of learning and memory, but the methodology represented by this finding launched the field into a new direction that was filled with exciting new possibilities. The ability to monitor a single neuron's activity in freely behaving animals could allow scientists to "see" for the first time learning and memory "on-line." Very soon afterwards it was revealed that the localized firing (or place field) of these "place cells" reflected not only the external sensory conditions but also the behavioral or movement state of the animal. That is, properties of an animal's movement (e.g. speed) induce changes in place fields, providing clear evidence that space per se is not the sole determinant of place cell discharge. These findings gave rise to the highly influential and strong hypothesis that the hippocampus generates a spatial cognitive map that incorporates not only the sensory qualities of an environment but also the spatial relationship among these features, and information that is relevant to an animal's behavior at different locations (O'Keefe and Nadel, 1978).

APPROACHES TO UNDERSTANDING THE MNEMONIC SIGNIFICANCE OF PLACE FIELDS

Since 1978, the difficult challenge has been, and continues to be, relating place fields to hippocampal-dependent learning and memory. Over the next 20-25 years, attempts to understand this relationship have taken a number of forms (see Mizumori et al. (in press) for a more detailed review).

1. Test whether experimental manipulations similarly affect learning and place fields. For example, NMDA antagonists are well known to block hippocampal-dependent learning (e.g., Morris et al., 1986), and NMDA blockade reduces the specificity or stability of place fields (e.g., Shapiro and Eichenbaum, 1999; McHugh et al., 1996).

2. Evaluation of the relationship between place fields and synaptic models of learning. The induction of long-term potentiation (a widely accepted model of neuroplasticity thought to reflect hippocampal learning mechanisms, Martin et al., 2000) has been shown to alter place fields (Dragoi et al., 2003).

*Correspondence to: Sheri J.Y. Mizumori, Psychology Department, Box 351525, University of Washington, Seattle, WA 98195-1525.

E-mail:mizumori@u.washington.edu

Accepted for publication 10 June 2006

DOI 10.1002/hipo.20209

Published online 31 July 2006 in Wiley InterScience (www.interscience. wiley.com).
3. Comparison of place fields across groups of animals with different learning capabilities. Place cells have been recorded from young and aged rats that often differ in their spatial learning abilities (Barnes et al., 1983; Mizumori et al., 1996; Tanila et al., 1997; Barnes et al., 1997; Wilson et al., 2004, 2005). The reported effects of aging on place fields are complex, as might be expected considering the aging process itself is complex, as is also the behavioral phenomenon under study.

4. Correlation between changes in place fields and time spent in a new environment. This approach has revealed that place fields initially develop quickly in new environments, but that changes continue to be observed for some time afterward (e.g., Wilson and McNaughton, 1993; Frank et al., 2004; Leutgeb et al., 2005, this issue; Frank et al., this issue). It should be noted that an assumption of this approach is that mere exposure to a new environment engages the same neural mechanisms that underlie hippocampal-dependent learning.

5. Characterization of place field responses to changes in familiar cues or cue arrangements. Place field reorganization (or remapping) is frequently reported to result from a variety of cue manipulations (e.g., O'Keefe, 1979; Young et al., 1994; Cressant et al., 1997; Shapiro et al., 1997; Tanila et al., 1997). These studies clearly show that the visual environment is a prominent factor determining place field stability. O'Keefe and Speakman (1987) showed that when cues were removed, place fields persisted. This was one of the first indication that memory modulates place fields. However, Knierim et al. and Leutgeb et al. (this issue) point out that not all place fields respond in the same way. CA1 place fields appear to respond differently from CA3 place fields. Furthermore, place cells within a single hippocampal subregion do not always respond in the same manner following experimental manipulation (Yeshenko et al., 2004; Anderson et al., this issue). That is, partial remapping is possible. That separate populations of place cells respond differently during the same recording session clearly indicates that we should not expect all place field responses to necessarily correspond with behavioral responses. This could certainly challenge efforts to understand how place fields reflect learned behaviors.

6. Testing the correlation between new learning and changes in place fields. Place fields have been shown to become more selective as rats learn a maze (Mizumori and Kalyani, 1997), and place fields may move toward new goal locations when animals are tested in a familiar 
room (e.g., Hollup et al., 2001; Lenck-Santini et al., 2001, 2002). Context-specific responses of place cells emerge when rats are required to distinguish between contexts (Smith and Mizumori, 2006, this issue). These data show that place fields can change in different ways during learning. However, as reflected in the comments by Kentros (this issue), this approach currently suffers from the difficulty of recording a large population of the same cells over an extended period of behavioral training. Also, caution must be taken to insure that behavioral differences during learning do not contribute to the place field changes that are observed.

7. Testing the effects of changes in cognitive demand, independent of differences in behavioral or sensory conditions. This approach has revealed that place fields are conditional upon recent or impending behavioral sequences of animals (e.g., Frank et al., 2000; Wood et al., 2000; Ferbinteanu and Shapiro, 2003), suggesting that place fields are modulated within a behaviorally relevant temporal (as well as spatial) domain. Furthermore, Yeshenko et al. (2001) showed that place fields respond to the retrieval of memories that support different cognitive strategies. In that study, a given cell was recorded as animals switched strategies within a single recording session. Thus, potential sensory, motor, or motivational confounds were eliminated. Similarly, Smith and Mizumori (2006) showed that changing the reward location within a single session induced dramatic place field reorganization. Again, sensory, behavioral, or motivational explanations could not account for the reorganization of place fields that was observed. Thus, it is becoming more clear that cognitive and reinforcement variables have dramatic impact on the spatial and temporal codes embodied by place fields.

\section{THE GOALS OF THIS SPECIAL ISSUE}

While the above approaches have resulted in a wealth of data that describe the regulation of plasticity of hippocampal place fields, a critical unanswered question is how these neural representations of space contribute to episodic memory functions of hippocampus. A 2005 Society for Neuroscience minisymposium (Hippocampal Place Fields and Episodic Memory) explicitly focused on this issue, and from this discussion emerged the present special issue in Hippocampus. The goal of the minisymposium was to not only review current research but to also discuss current issues and future directions for this field. These goals are mirrored in this special issue. One important feature of the minisymposium was that relatively new investigators to this field took the lead to present current data relevant to some of the most pressing issues in this field. Similar to the minisymposium, relatively "junior" investigators of place cells and learning and memory have taken the lead in the articles of the current special issue.

One way to assess how far we, as a research field, have come is to consider where we were in 1999 when the first special issue on hippocampal place cells appeared in Hippocampus. That issue contained articles that addressed fundamental questions regarding the nature of the sensory and spatial coding by hippocampal neurons. In their introductory remarks, Lynn Nadel and Howard Eichenbaum noted the emergence of a view that hippocampal place cells represent spatial context information. It was further suggested that understanding how spatial context processing contributes to episodic memory will likely require an expanded consideration of adjacent connected regions such as the subiculum and entorhinal cortex (EC) (Sharp, 1999), as well as a more detailed comparison of subregions within hippocampus (e.g., CA1 vs. CA3 regions; Mizumori et al., 1999). Indeed, in many respects, the present special issue takes off where the previous special issue ended. New technological advances have led to more sophisticated theories about how hippocampal neurons integrate spatial and temporal information. Novel learning paradigms have offered new potential for more directly testing the relationship between place fields and learning or memory functions. With these tools, the articles targeted key components of episodic memory for analysis.

\section{A COMPONENT ANALYSIS OF THE ROLE OF PLACE FIELDS IN EPISODIC MEMORY}

Episodic memory, almost by definition, is a complex and multifaceted cognitive function comprised of many components. Therefore, in an effort to understand how place fields relate to hippocampal-dependent episodic memory, recent studies have taken a component analysis approach to assess the basic question of whether place fields are part of a neural code that could be used to create or retrieve episodic memories. Specifically, the articles contained in this issue focus on how place fields relate to context coding, temporal processing, rapid learning, and stable representation.

Context coding. Episodic memory refers to a memory system within which we remember specific and unique events in our lives (Tulving, 2002). Events are often defined according to the perceived context of a situation. Ferbinteanu et al., Smith and Mizumori, Anderson et al., and Leutgeb et al. (in different articles within this issue) explore various aspects of context coding by hippocampal place cells. Their findings provide evidence that support the view that hippocampus encodes context-specific information. In addition, these papers, and others, take on the tough issue of defining what is meant by the term "context," and the issue of whether spatial context analysis is a special case of general context processing, or vice versa. Although these papers present very thoughtful discussions about how hippocampal context representations are related to episodic memory, this issue remains unresolved, requiring additional discussion and analysis.

Temporal processing. Recall of events involves not only the identification of specific items within a particular place but also changes in these items or places as a function of time. O'Keefe and Nadel (1978) expressed that the addition of a temporal component changes "the basic spatial map into a human episodic memory system." Thus, any neurobiological model of episodic memory should include not only neural codes for contextual information but also a neurobiological mechanism for linking contexts or changes in contexts across time (see discussion in Ferbinteanu et al., this issue). O'Keefe and Recce (1993) provided the first evidence that place fields can be organized along a 
temporal dimension by demonstrating that the first spike in a complex-spike burst by a place cell occurs at progressively earlier phases of the ongoing theta cycle, a phenomenon referred to as phase precession. This finding is significant because it suggested a mechanism for linking temporally extended behavior of animals with the comparatively rapid synaptic plasticity mechanisms that are thought to underlie learning (Skaggs et al., 1996; Buzsaki, 2005; Zugaro et al., 2005; Kentros, this issue; Maurer et al., this issue).

Temporal coding by hippocampal neurons has also been analyzed by studying place field responses relative to temporally ordered sequences of behavioral activity. This is illustrated by the conditional place fields described by de $\mathrm{Hoz}$ and Wood (this issue) and Ferbinteanu et al. (this issue). These fields were expressed in relationship to future or recent behavioral acts. Therefore, these data were taken as evidence for retrospective and prospective coding by place fields, a function that may reflect the organization of contextual information along a temporal dimension (also see Leutgeb et al., Maurer et al., and Frank et al. for further discussion). Manns and Eichenbaum (this issue) summarize findings that suggest that hippocampal unit activity represents different phases of a sequence learning task, which is also consistent with the view that hippocampal neural codes may be involved in learned sequences of behavior.

Even though the study of temporal processing by place cells has been approached from different perspectives, it remains diffcult to see how the described temporal properties are sufficient to account for the sort of "mental time travel" attributed to human episodic memory (Tulving, 2002). Some encouragement on this issue was recently provided by Foster and Wilson (2006) who showed that hippocampal place cells of awake animals "replay" their activity pattern in reverse temporal sequence in between bouts of active exploration. It remains to be determined whether the degree or clarity of reverse replay is predictive of behavioral learning or the accurate expression of memory. Even if the latter is found to be true, another related and more difficult issue is to determine whether rodents have an autonoetic consciousness that allows one to be aware of the subjectively experienced time in which events occur (Tulving, 2002), another critical element of human episodic memory.

Rapid learning. In addition to processing contextual information across time, episodic memories are typically acquired rapidly. To evaluate the neural mechanisms that underlie this component of an episodic memory system, a number of articles described studies that test place field responses to novel environment (see articles by Anderson et al., Knierim et al., Frank et al., and de Hoz and Wood). It seems that place fields change or emerge rapidly upon initial exposure to a new environment, and in some cases continue to change for a time afterward.

Long term stability. Episodic memories are thought to be enduring. Therefore, studies examining factors that contribute to the long term stability of place fields are important. Kentros and Knierim et al. (this issue) discuss data relevant to this issue, as well as the technical challenges associated with long term recordings of the single unit activity. These data and issues are worthy of continued investigations, regardless of whether one views hippocampus as a memory storage device, or a structure that enables memory consolidation in efferent brain regions.

\section{THE CHALLENGES BEFORE US}

Over the decades, we have learned a tremendous amount about the dynamic properties of place fields, and how they relate to changes in the external environments, task demands, and an animal's behavioral responses. However, numerous issues remain to be resolved if we are to understand the role for place representation in episodic memory. Some of these were initially raised by Nadel and Eichenbaum (1999) in their special issue on place cells, but have evolved as new findings have emerged.

Although place cells clearly integrate spatial and nonspatial information, the location of an animal remains perhaps the strongest, most consistent predictor of cell discharge across the different laboratories. Thus, consistent with the suggestion by Nadel and Wilner (1980) some 25 years ago, it appears that place fields represent spatial contextual information. That is, nonspatial information may be organized according to a spatial reference frame, at least in navigating animals (see below for further discussion). The articles in this special issue further demonstrate that place field responses are consistent with the view that hippocampus contributes to key elements of episodic memory, such as spatial context analysis, temporal organization and rapid encoding of contextual information, and perhaps long term stability of mnemonic representations. Precisely how these neuronal responses become integrated to contribute to episodic memory, however, continues to be a significant challenge. Furthermore, as pointed out by de Hoz and Wood (this issue) and Smith and Mizumori (this issue), these elemental processes are also important for other kinds of memory systems, such as semantic memory. Therefore, we are still in search for neurophysiological evidence that hippocampus makes a unique contribution to episodic memory. This goal is made more complicated by the possibility that hippocampal processing is automatic and continuous (e.g., Morris and Frey, 1997; Mizumori et al., 1999), regardless of the hippocampal-dependent nature of the task (Yeshenko et al., 2004). Perhaps a more direct link to episodic memory will become clearer once we resolve basic issues such as how to identify and quantify place fields (Maurer et al. and Kentros, this issue), how best to link unit activity and memory functions (e.g., according to the type of behavioral correlate or changes in firing rates, Kentros, this issue), and how temporal and spatial aspects of cell discharge relate to one another during hippocampal-dependent learning. These fundamental issues should be placed within an evolutionarily meaningful context (e.g., Manns and Eichenbaum, and Knierim et al., this issue), and the behavioral tasks will need to selectively engage episodic functions so that episodic memory can be quantified and then directly related to neural activity.

In addition to the set of outstanding somewhat broad issues listed above, there are many specific challenges. These are grouped below according to three basic questions. They are not necessarily mutually exclusive. 
1. How is place field information used in the formation and retrieval of episodic memories? To answer this broad question, we must understand more clearly how place fields are formed in the first place. O'Keefe and Nadel (1978) argued that nonspatial information becomes embedded within a spatial reference (i.e., a cognitive map), but there was no strong empirical evidence to support that view, until recently. Hafting et al. (2005) reported that the cortical afferent structure for hippocampal place cells, the EC, contains "grid cells" whose place fields define the vertices of a two-dimensional tessellating triangular grid. The stability of these grids across different environments suggests that EC may provide hippocampus with a spatial reference frame within which to organize nonspatial data. This may account for the prominence of place fields during active exploration. Perhaps activation of the theta rhythm as occurs during exploration engages EC grid representations. This hypothesis is consistent with the suggested important role of exploration in the generation of a cognitive map (O'Keefe and Nadel, 1978; see further discussion in Smith and Mizumori, Anderson et al., and Leutgeb et al., this issue). We may now ask more detailed questions about the role of exploration. For example, is movement speed related to more accurate or stable grid representations? Notably, rats do tend to move about more slowly during initial exposures to a new situation, and response latency declines after learning has occurred.

To understand the role of place fields in episodic memory, we must also determine the functional organization of memory processes within hippocampus. To accomplish this, a number of issues need to be resolved, such as whether spatial context is a special case of a general context processing function. An answer to this question depends in part on our understanding of how spatial context information is used. Adding to a suggestion by O'Keefe and Nadel (1978), we (Mizumori et al., 1999, 2000; Smith and Mizumori, 2006, this issue) hypothesized that hippocampus uses spatial context information to engage in a matchmismatch operation. The output of this operation can be used to inform the broader episodic memory circuitry of changes in the current task situation. Since animals can detect changes in task situations independent of their own movement, it was argued that context analysis can proceed independent of exploration-induced spatial maps. Therefore, the neuroarchitecture of hippocampus may function continuously as a context comparator. During exploration, the predominant mode of context analysis becomes spatial.

Understanding the functional organization of hippocampal memory processes requires that we understand better the significance of CA1 and CA3 differences in place field specificity and sensitivity to context changes (Leutgeb et al. and Knierim et al., this issue). For example, what is the hierarchical nature of information coding between hippocampal subregions, and also within a single area (discussed in Smith and Mizumori, and Maurer et al., this issue)?

Another specific issue to resolve is whether place fields have different roles during new learning (i.e., consolidation) relative to retrieval of learned information. We have evidence that place fields change during new learning and exposure to new environments (see articles by Anderson et al., Kentros, Frank et al., and Knierim et al., this issue), and there is some evidence that place fields change during retrieval of different memories (e.g., Smith and Mizumori, this issue; Yeshenko et al., 2001). We do not yet, however, fully understand how these changes in place field characteristics relate to specific cognitive operations (e.g., encoding, retrieval, etc.).

2. Do place field responses map onto behavioral performance? If so, how? If not, why not? A number of studies have found that changes in place fields do not necessarily predict a corresponding change in behavior (e.g., Cooper and Mizumori, 2001; Jeffery et al., 2003; Frank et al., this issue). Moreover, since CA1 and CA3 place fields respond differently to experimental manipulations (e.g., Leutgeb et al. and Knierim et al., this issue), and since partial reorganization is often observed within the same recording session following a change in context (e.g., Mizumori et al., 1999; Anderson et al., this issue), it seems unlikely to expect that changes in place fields directly implies a change in behavior. On the other hand, if behavior is altered by a manipulation, one may expect to observe place field changes. The asymmetry in this relationship could be due, for example, to the availability of different strategies during task performance. Thus if one neural circuit (involving place cells) changes, other circuitry may functionally compensate, resulting in no observable alteration in behavior. On the other hand, if a treatment impairs learning, then almost by definition, not only is the hippocampal circuit affected but so would be the compensatory circuit.

The findings of partial reorganization of place fields following a variety of experimental manipulations suggest that hippocampus represents not only the current context, but also information based on an animal's expectations, or memory. More specifically, the persistent neural codes could reflect pattern completion or memory retrieval processes. Partial reorganization has been interpreted as being consistent with the view that hippocampus uses contextual information to perform a match-mismatch comparison to determine whether the context is different from what is expected based on past experience (e.g., Mizumori et al., 1999; Lisman, 1999; Smith and Mizumori, and Anderson et al., this issue). This mechanism predicts that hippocampus would be selectively and differentially activated during novel presentation of cues, or when there is a change in familiar cues.

From a neural systems perspective, it is also desirable to understand how hippocampal place field information exerts influences over behavioral control systems of the brain such as the basal ganglia or frontal cortex. Does the hippocampus provide important contextual information that guides control over behavior? Recent findings of context-dependent place cells in striatum (Mizumori et al., 1999; Yeshenko et al., 2004) suggest that the answer is yes.

3. How do hippocampal place fields function within the broader neural system that underlies episodic memory? Many of the articles in this special issue stressed the importance of taking a broad neural systems perspective when trying to understand the significance of place fields. In the narrowest sense, one could ask how hippocampal afferent and efferent systems are related to place cell functions. The value of this approach can be appreciated when one considers the almost immediate impact that findings of EC grid cells (Hafting et al., 2005) and correlated firing between hippocampus and prefrontal cortex (Siapas et al., 2005) 
have had in our conceptualization of the significance of place fields. In both cases, our view of the significance of place fields has become more refined and focused.

A second level of neural systems analyses is one in which we seek to understand how hippocampal place fields relate to brain areas more distant than a single synapse away. As an example, Manns and Eichenbaum (this issue) provide a clear description of the known anatomical connections through which spatial and nonspatial information arrives in EC. Delineation of such pathways makes predictions about the functional contributions of, for example, parietal cortex to place fields. Additionally, as mentioned above, understanding how hippocampal place cells impact ongoing behavior will likely require understanding how response selection circuitry (involving midbrain and forebrain structures) is modulated by hippocampal output.

A third level of neural systems consideration involves taking an evolutionary perspective (Mann and Eichenbaum, and Knierim et al., this issue). With this approach, natural behaviors are observed in natural settings in hopes that the functional significance of hippocampal spatial codes will become more clear. Furthermore, this approach could allow for comparisons of place fields across different species with different ecological demands. A question is whether place fields could be adapted to serve different functions depending upon an animal's ecological specializations.

In the past ten years or so, our understanding of the dynamic properties of place fields has moved forward in important ways. Greater technological and theoretical sophistication has allowed us to ask more detailed questions about temporal and spatial aspects of place fields. Many laboratories have begun to address the difficult issue of the significance of place fields for hippocampal-dependent learning and memory by recording place cells during relevant learning tasks. There is also increasing effort to take a neural systems perspective so that we may understand the neural context within which hippocampal place cells function. As a result of these advances, we are beginning to have a better understanding of what information is represented by place fields, as well as the conditions under which this what information is expressed. At the same time, we continue to be challenged to understand how place field information contributes specifically to hippocampal-dependent learning, and more generally to broader memory systems such as episodic memory. With continued exponential growth in terms of both technological advances and the degree of sophistication of behavioral paradigms, an answer to the how question may be just around the bend.

Sheri J.Y. Mizumori University of Washington Seattle, Washington

\section{REFERENCES}

Barnes CA, McNaughton BL, O’Keefe J. 1983. Loss of place specificity in hippocampal complex spike cells of senescent rat. Neurobiol Aging 4:113-119.
Barnes CA, Suster MS, Shen J, McNaughton BL. 1997. Multistability of cognitive maps in the hippocampus of old rats. Nature 388:272275.

Buzsáki G. 2005. Theta rhythm of navigation: Link between path integration and landmark navigation, episodic and semantic memory. Hippocampus 15:827-840.

Cooper BG, Mizumori SJY. 2001. Temporary inactivation of retrosplenial cortex causes a transient reorganization of spatial coding in hippocampus. J Neurosci 21:3986-4001.

Cressant A, Muller RU, Poucet B. 1997. Failure of centrally placed objects to control the firing fields of hippocampal place cells. J Neurosci 17:2531-2542.

Dragoi G, Harris KD, Buzsáki G. 2003. Place representation within hippocampal networks is modified by long-term potentiation. Neuron 39:843-853.

Ferbinteanu J, Shapiro ML. 2003. Prospective and retrospective memory coding in the hippocampus. Neuron 40:1227-1239.

Foster DJ, Wilson MA. 2006. Reverse replay of behavioural sequences in hippocampal place cells during the awake state. Nature 440:680-683.

Frank LM, Brown EM, Wilson MA. 2000. Trajectory encoding in the hippocampus and entorhinal cortex. Neuron 27:169-178.

Frank LM, Stanley GB, Brown EN. 2004. Hippocampal plasticity across multiple days of exposure to novel environments. J Neurosci 24: 7681-7689.

Hafting T, Fyhn M, Molden S, Moser MB, Moser EI. 2005. Microstructure of a spatial map in the entorhinal cortex. Nature 436:801-806.

Hollup SA, Molden S, Donnett JG, Moser MB, Moser EI. 2001. Accumulation of hippocampal place fields at the goal location in an annular watermaze task. J Neurosci 21:1635-1644.

Jeffery KJ, Gilbert A, Burton S, Strudwick A. 2003. Preserved performance in a hippocampal-dependent spatial task despite complete place cell remapping. Hippocampus 13:175-189.

Kuhn T. 1970. The Structure of Scientific Revolutions, 2nd ed. Chicago: University of Chicago Press.

Lenck-Santini PP, Save E, Poucet B. 2001. Evidence for a relationship between place-cell spatial firing and spatial memory performance. Hippocampus 11:377-390.

Lenck-Santini PP, Muller RU, Save E, Poucet B. 2002. Relationships between place cell firing fields and navigational decisions by rats. J Neurosci 22:9035-9047.

Leutgeb S, Leutgeb JK, Barnes CA, Moser EI, McNaughton BL, Moser MB. 2005. Independent codes for spatial and episodic memory in hippocampal neuronal ensembles. Science 309:619-623.

Lisman JE. 1999. Relating hippocampal circuitry to function: Recall of memory sequences by reciprocal dentate-CA3 interactions. Neuron 22:233-242.

Martin SJ, Grimwood PD, Morris RG. 2000. Synaptic plasticity and memory: An evaluation of the hypothesis. Annu Rev Neurosci 23: 649-711.

McHugh TJ, Blum KI, Tsien JZ, Tonegawa S, Wilson MA. 1996. Impaired hippocampal representation of space in CA1-specific NMDAR1 knockout mice. Cell 87:1339-1349.

Mizumori SJY, Kalyani A. 1997. Age and experience-dependent representational reorganization during spatial learning. Neurobiol Aging 18:651-659.

Mizumori SJY, Lavoie AM, Kalyani A. 1996. Redistribution of spatial representation in the hippocampus of aged rats performing a spatial memory task. Behav Neurosci 110:1006-1016.

Mizumori SJY, Ragozzino KE, Cooper BG, Leutgeb S. 1999. Hippocampal representational organization and spatial context. Hippocampus 9: 444-451.

Mizumori SJY, Cooper BG, Leutgeb S, Pratt WE. 2000. A neural systems analysis of adaptive navigation. Mol Neurobiol 21:57-82.

Mizumori SJY, Smith DM, Puryear CB. Mnemonic contributions of hippocampal place cells. In: Martinez JL Jr, Kesner RP, editors. Neurobiology of Learning and Memory. San Diego, CA: Academic Press (in press). 
Morris RG, Frey U. 1997. Hippocampal synaptic plasticity: Role in spatial learning or the automatic recording of attended experience? Phil Trans R Soc Lond B Biol Sci 352:489-503.

Morris RGM, Anderson E, Lynch GS, Baudry M. 1986. Selective impairment of learning and blockade of long-term potentiation by an $N$ methyl-D-aspartate receptor antagonist, AP5. Nature 319:774-776.

Nadel L, Eichenbaum H. 1999. Introduction to the special issue on place cells. Hippocampus 9:341-345.

Nadel L, Wilner J. 1980. Context and conditioning: A place for space. Physiol Psychol 8:218-228.

O'Keefe J. 1979. A review of the hippocampal place cells. Prog Neurobiol 13:419-439.

O’Keefe J, Dostrovsky J. 1971. The hippocampus as a spatial map. Preliminary evidence from unit activity in the freely moving rat. Brain Res 34:171-175.

O'Keefe J, Nadel L. 1978. The Hippocampus as a Cognitive Map. Oxford: Oxford University Press.

O’Keefe J, Recce ML. 1993. Phase relationship between hippocampal place units and the EEG theta rhythm. Hippocampus 3:317-330.

O'Keefe J, Speakman A. 1987. Single unit activity in the rat hippocampus during a spatial memory task. Exp Brain Res 68:1-27.

Shapiro ML, Eichenbaum H. 1999. Hippocampus as a memory map: Synaptic plasticity and memory encoding by hippocampal neurons. Hippocampus 9:365-384.

Shapiro ML, Tanila H, Eichenbaum H. 1997. Cues that hippocampal place cells encode: Dynamic and hierarchical representation of local and distal stimuli. Hippocampus 7:624-642.

Sharp PE. 1999. Complimentary roles for hippocampal versus subicular/ entorhinal place cells in coding place, context, and events. Hippocampus 9:432-443.

Siapas AG, Lubenov EV, Wilson MA. 2005. Prefrontal phase locking to hippocampal theta oscillations. Neuron 46:141-151.

Skaggs WE, McNaughton BL, Wilson MA, Barnes CA. 1996. Theta phase precession in hippocampal neuronal populations and the compression of temporal sequences. Hippocampus 6:149-172.
Smith DM, Mizumori SJY. 2006. Learning-related development of context-specific neuronal responses to places and events: The hippocampal role in context processing. J Neurosci 26:31543163.

Tanila H, Shapiro ML, Eichenbaum H. 1997. Discordance of spatial representation in ensembles of hippocampal place cells. Hippocampus 7:613-623.

Tulving E. 2002. Episodic memory: From mind to brain. Annu Rev Psychol 530:1-25.

Wilson IA, Ikonen S, Gureviciene I, McMahan RW, Gallagher M, Eichenbaum H, Tanila H. 2004. Cognitive aging and the hippocampus: How old rats represent new environments. J Neurosci 24:38703878.

Wilson IA, Ikonen S, Gallagher M, Eichenbaum H, Tanila H. 2005. Age-associated alterations of hippocampal place cells are subregion specific. J Neurosci 25:6877-6886.

Wilson MA, McNaughton BL. 1993. Dynamics of the hippocampal ensemble code for space. Science 261:1055-1058.

Wood ER, Dudchenko PA, Tobitsek RJ, Eichenbaum H. 2000. Hippocampal neurons encode information about different types of memory episodes occurring in the same location. Neuron 27:623-633.

Yeshenko O, Guazzelli A, Mizumori SJY. 2001. Differential hippocampal neuronal activity during place or response performance on a T-maze. Soc Neurosci. 2001 Abstract Viewer and Itinerary Planner.

Yeshenko O, Guazzelli A, Mizumori SJY. 2004. Context-dependent reorganization of spatial and movement representations by simultaneously recorded hippocampal and striatal neurons during performance of allocentric and egocentric tasks. Behav Neurosci 11:751769.

Young BJ, Fox GD, Eichenbaum H. 1994. Correlates of hippocampal complex-spike cell activity in rats performing a nonspatial radial maze task. J Neurosci 1:6553-6563.

Zugaro MB, Monconduit L, Buzsaki G. 2005. Spike phase precession persists after transient intrahippocampal perturbation. Nat Neurosci 8: $67-71$. 